\title{
Reduction of an in vitro Intraradicular Multispecies Biofilm Using Two Rotary Instrumentation Sequences
}

\author{
Angelo Zavattini ${ }^{1} \quad$ Jonathan Cowie ${ }^{2} \quad$ Sadia Niazi ${ }^{1}$ \\ Federico Foschi ${ }^{1,3}$ \\ ${ }^{1}$ Department of Endodontics, Faculty of Dentistry, Oral \& \\ Craniofacial Sciences, Postgraduate Centre, Guy's Hospital, London \\ Bridge, King's College London, London, United Kingdom \\ ${ }^{2}$ Circus Dental Practice, Bath, United Kingdom \\ ${ }^{3}$ Department of Therapeutic Dentistry, I.M. Sechenov First Moscow \\ State Medical University, Moscow, Russia \\ ${ }^{4}$ Departamento de Odontologia, Facultad de Ciencias de la Salud, \\ Universidad CEU Cardenal Herrera, Valencia, Spain
}

Massimo Giovarruscio ${ }^{1,3}$ Salvatore Sauro 3,4

\begin{abstract}
Address for correspondence Angelo Zavattini, DDS, PhD, Department of Endodontics, Faculty of Dentistry, Oral \& Craniofacial Sciences, King's College London, Postgraduate Centre, Floor 22, Tower Wing, Guy's Hospital, London Bridge, London SE1 9RT, United Kingdom (e-mail: angelo.zavattini@kcl.ac.uk).
\end{abstract}

Eur J Dent 2020;14:1-7

\begin{abstract}
Keywords

- biofilm

- chemomechanical preparation

- confocal microscope

- endodontic infection

- ProTaper Gold

- RaCe

- root canal

Objective The purpose of this research was to investigate the effect per se of two shaping and cleaning techniques on the reduction of an in vitro multispecies biofilm.

Materials and Methods A total of 39 freshly extracted monoradicular teeth for periodontal reason were decoronated. Roots were sectioned longitudinally. After autoclaving, a specific stressed biofilm was grown on the root halves that were subsequently reassembled in a silicone index. Two treatments ( $n=9$ each)-RaCe (Schottlander; Letchworth Garden City, United Kingdom) and ProTaper Gold (PTG; Dentsply Maillefer, Baillagues, Switzerland)-were tested; three noninstrumented samples served as a control group and three were rinsed with saline. Posttreatment samples were taken at three different levels of the root. Colony-forming units were counted after incubations. Additionally, three treatments ( $n=5$ each)-RaCe, PTG, and saline only-were evaluated under a confocal laser scanning microscope (CLSM).

Statistical Analysis Statistical analysis was conducted using Tukey's test and analysis of variance to evaluate the post-instrumentation bioburden.

Results Both instrumentations were able to reduce the biofilm; however, differences were not present between them $(p>0.05)$. CLSM showed biofilm killing and disruption through mechanical shaping alone.

Conclusions Intraradicular biofilm is reduced with mechanical shaping. There was no difference between RaCe and PTG systems in biofilm reduction despite differences in design, file sequence, and rotational speed.
\end{abstract}

\section{Introduction}

It is well understood that there is a causal relationship between the presence of bacteria and apical periodontitis. ${ }^{1-3}$ The clinical objective of root canal treatment is to reduce the number of microorganisms to a level that can enable periapical healing. ${ }^{4-6}$ Chemomechanical preparation is undertaken with a combination of instrumentation and irrigation. The taper of the endodontic space should be adequate to permit irrigants to access the apical and lateral extents of the complex endodontic anatomy. ${ }^{7,8}$ By consequence, it is clear how the characteristics of endodontic rotary instrument play an important role in the clinical success.

Bacteria exist inside the endodontium mostly as biofilm. ${ }^{9}$ There are two main features of biofilms that are of particular importance in relation to endodontic infections. First, there is an augmented tolerance to antibiotic by bacterial biofilm, and, second, through altered phenotype and genotype, the virulence of bacteria within a biofilm is greater. Biofilms are 
considered to be less susceptible to antimicrobial killing than planktonic bacteria. ${ }^{10}$ Single- and multispecies biofilms have previously been predictably grown in extracted single-rooted teeth to assess bacterial reduction when using ultrasonics and irrigants. ${ }^{11}$

Rotary nickel-titanium (NiTi) files are a mainstay of contemporary endodontic instrumentation. ${ }^{12}$ The properties of NiTi files enable optimal canal shaping or obturation using the current obturation techniques available. ${ }^{13}$ Mechanically, NiTi files should be resistant to wear and have good cutting efficiency, and this relates to the hardness of the file. Good torsional properties improve resistance to fracture, and resistance to deformation improves centering within the canal, thus reducing iatrogenic mistakes. ${ }^{14,15}$

The recently introduced RaCe (Reamer with Alternating Cutting Edge) file (Schottlander; Letchworth Garden City, United Kingdom) has a noncutting tip and a triangular cross-section. Alternating cutting edges avoid the screwing effect and have the advantage of operating with extremely low torque. The RaCe files therefore have the capability to reduce "dragging in" to the root canal. ${ }^{15}$ RaCe files also undergo electro-polishing after a machining process to improve torsional resistance and fatigue resistance. The RaCe 123 files are devised as a three-instrument sequence: T1, $10 / .06 ; \mathrm{T} 2,25 / .05$, and T3, 35/.04.

The purpose of this in vitro research was to compare the ability of the RaCe instruments with that of a well-established instrumentation system (ProTaper Gold; Dentsply Maillefer, Baillagues, Switzerland) on the removal of an in vitro nutrient-stressed endodontic multispecies biofilm ${ }^{16}$ by means of quantitative viable counts and confocal laser scanning microscope (CLSM). The null hypothesis was that no difference was present between the effect on mature endodontic biofilms of the RaCe 123 and ProTaper Gold instrumentation.

\section{Materials and Methods}

The study was conducted in compliance with the principles of the Declaration of Helsinki and Good Clinical Practice after approval from the London Westminster Research Ethics Committee (Research Ethics Committee Reference Number 10/H0804/056). Patient information sheets were distributed, and informed written consent obtained prior to study commencement.

A total of $39(n=39)$ monoradicular teeth were extracted for the study.

\section{Specimen Preparation}

The teeth were decoronated and the working lengths standardized to $15 \mathrm{~mm}$, after which the specimens were mechanically instrumented following a clear protocol. First, every canal was scouted and deemed patent using an ISO (International Organization for Standardization) size $10 \mathrm{~K}$-Flexofile (Dentsply Maillefer). The samples were then enlarged up to a size $20 \mathrm{~K}$-Flexofile. Specimens were then sectioned longitudinally through the root canal using a 0.3-mm-thick diamond saw blade (Extec; Enfield,
Connecticut, United States) on an 11 to 1,180 IsoMet lowspeed saw (Buehler, Düsseldorf, Germany). Roots were reapproximated and positioned into a block of mixed silicone impression material (Aquasil; Dentsply DeTrey, Konstanz, Germany) in a glass case jar, ensuring that the coronal end of the prepared root was flushed with the surface of the silicone block. After setting of the silicone compound, the impression material and the root were marked so that the orientation of the root in the block could be verified. A sequential number was allocated to the root and the silicone block so that they could be easily identified. ${ }^{11}$

\section{Biofilm Development}

The emi roots were detached from the impression material and rinsed in a $17 \%$ solution of EDTA (ethylenediaminetetraacetic acid) for 1 minute to remove the smear layer and were then again rinsed thoroughly with phosphate-buffered saline. Both root parts were then sterilized individually at $121^{\circ} \mathrm{C}$ for 15 minutes. The sample with the most even canal and fully including the apex was used for culturing the selected microorganism.

After the sterilizing process, nutrient-stressed biofilms composed of Propionibacterium acnes, Staphylococcus epidermidis, Actinomyces radicidentis, Streptococcus mitis recovered as predominant taxa from persistent endodontic infections, ${ }^{17}$ and Enterococcus faecalis strain OMGS 3202 present in refractory lesions ${ }^{18}$ were cultured on the root half for 14 days using a defined protocol. ${ }^{16}$ The burdens were routinely cultured anaerobically at $37^{\circ} \mathrm{C}$ in MACS MG-1000 anaerobic workstation ( $80 \%$ nitrogen, $10 \%$ hydrogen, $10 \%$ carbon dioxide) on Fastidious Anaerobe Agar (Lab M, Heywood, United Kingdom) enhanced with 5\% defibrinated horse blood. Initial strains were set up in filter-sterilized modified fluid universal medium (mFUM) incubated anaerobically at $37^{\circ} \mathrm{C}$ for 3 hours. The absorbance was regulated with fresh mFUM to 0.5 at $540 \mathrm{~nm}$ (iEMS Reader MF, Labsystems, Basingstoke, United Kingdom). Every specimen was then placed in a 24-well tray and seeded with $400 \mu \mathrm{L}$ each $\left(\sim 10^{7} \mathrm{CFU} / \mathrm{mL}\right)$ of the five initial strains. The microorganisms were grown anaerobically for 7 days with regular medium change every 24 hours. Afterward, inoculated specimens were maintained for further 7 days in the anaerobic cabinet without medium replenishing to induce a starvation/resistance phase in the biofilms.

\section{Instrumentation and Sampling for Quantitative Viable Counts}

The infected root halves were reassembled into the silicon index to allow root canal shaping. Twenty-four specimens were divided into four groups. In experimental group A $(n=9)$, root canals were shaped using the RaCe 123 instruments; T1, T2 instruments were used in sequence on a 6:1 contra-angle powered by an X-Smart Plus Endodontic Motor (Dentsply Maillefer) at $600 \mathrm{rpm}$. In experimental group B $(n=9)$, root canal preparation using ProTaper Gold, and S1, S2, F1, and F2 instruments were used in sequence 
with the same motor at $300 \mathrm{rpm} .{ }^{19}$ In saline group C ( $\left.n=3\right)$, standard irrigation was performed with $6 \mathrm{~mL}$ of solution for 2 minutes using a 27-gauge side-vented irrigating needle and 3-mL syringe (Monoject, Tyco Healthcare, Gosport, United Kingdom). In control group D $(n=3)$, no root canal preparation was done.

After instrumentation, the specimens were disassembled and the postinstrumentation samples of the canal content were obtained using sterile paper points (Dentsply DeTrey, Konstanz, Germany). The samples were obtained from the coronal, middle, and apical thirds of the root canal by prior markings with a scalpel blade at 3,6 , and $9 \mathrm{~mm}$ from the coronal end of the root canal, respectively.

Colony-forming units (CFUs) were determined using a standardized laboratory procedure.

\section{Confocal Laser Scanning Microscope}

Fifteen multispecies biofilms were grown on the tooth models and randomly divided into three treatment groups: group A (RaCe 123) $(n=5)$, group B (ProTaper) $(n=5)$, and group $C$ (saline), as described above. The chosen root halves were marked with live/dead BacLight Bacterial Viability Kit (Invitrogen, United Kingdom) before as well as after instrumentation. The stained viable cells were detected using a CLSM, and the biofilm structure was analyzed according to a previous study protocol. ${ }^{20}$ The preoperative images constituted the internal control of the developed biofilm.

\section{Statistical Analysis}

Data were analyzed using SPSSv.19 (IBM Corp., Chicago, Illinois, United States). Data were normalized prior to statistical evaluation and then analyzed using analysis of variance and the Tukey HSD (honestly significant difference) test. The significance level was set at $\alpha=0.05$.

\section{Results}

The residual bacterial CFUs were detected in all three areas of the root canal (coronal, middle, and apical third) after instrumentation ( $\boldsymbol{- T a b l e} \mathbf{1}$ ). Differences between the CFUs at the three root canal levels were not statistically significant $(p>0.05)$. Both instrumentation techniques significantly reduced the number of bacteria compared with the controls and saline with conventional syringe irrigation $(p<0.05)$. No significant difference was detected between the two instrumentation approaches $(p>0.05)$ ( - Fig. 1). Both were equally effective in reducing the bacterial counts compared with the controls and saline group at all levels of the root canal space.

CLSM image taken before the respective instrumentation with Race 123 and ProTaper Gold served as the internal controls. According to the CLSM results, both Race 123 and ProTaper Gold were significantly effective in bacterial killing, with $63 \%$ for Race 123 and $62 \%$ for ProTaper Gold), as compared with their internal controls $(p<0.05)(-$ Fig. 2A). They were also effective in biofilm disruption as shown by a significant reduction in their mean percent substratum coverage as rivaled to the internal control ( - Fig. 2B). Nevertheless, no differences could be seen between the bacterial killing and biofilm disruption efficacy between these two. Saline used with conventional syringe irrigation also reduced the substratum coverage when compared with the uninstrumented controls, however less than that of Race 123 and ProTaper

\section{CFU reduction normalized to Control}

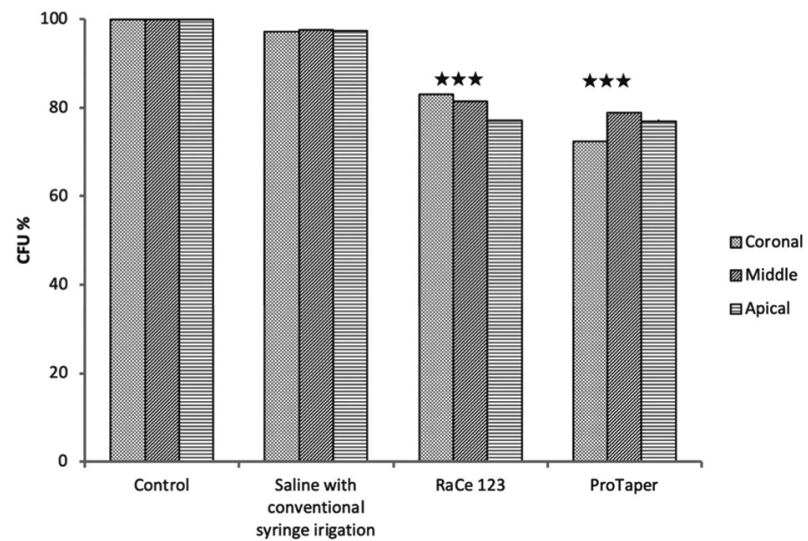

Fig. 1 Bar chart depicting the relative colony-forming unit (CFU) reduction at the coronal, middle, and apical positions normalized to the control group presented. There was no statistically significant difference $(p>0.05)$ between the CFUs at the three root canal levels. Both instrumentation techniques significantly reduced the number of bacteria compared with the controls and saline group $(p<0.05)$ (asterisks). No significant difference was detected between the two instrumentation approaches $(p>0.05)$.

Table 1 Quantitative viable counts of the two different instrumentation techniques on the in vitro nutrient-stressed multispecies biofilm model

\begin{tabular}{|l|l|l|l|}
\hline \multirow{2}{*}{ Experimental groups } & \multicolumn{4}{l|}{ Microbial counts as $\log _{10}$ per sample } \\
\cline { 2 - 4 } & Coronal, mean \pm SE & Middle, mean \pm SE & Apical, mean \pm SE \\
\hline Control & $6.74(0.10)$ & $6.61(0.07)$ & $6.56(0.08)$ \\
\hline Saline with conventional syringe irrigation & $6.55(0.02)$ & $6.45(0.07)$ & $6.39(0.03)$ \\
\hline RaCe 123 & $5.59(0.14)^{\mathrm{a}}$ & $5.39(0.06)^{\mathrm{a}}$ & $5.06(0.01)^{\mathrm{a}}$ \\
\hline ProTaper & $4.88(0.05)^{\mathrm{a}}$ & $5.22(0.03)^{\mathrm{a}}$ & $5.05(0.32)^{\mathrm{a}}$ \\
\hline
\end{tabular}

Abbreviation: SE, standard error.

aValues significantly less than the controls and saline group at the same sampling sites $(p<0.05)$. 
4 Reduction of Intraradicular Multispecies Biofilm Zavattini et al.

(-Fig. 2B). Moreover, saline did not show any significant bacterial killing effect with $14 \%$ as compared with Race 123 $(63 \%)$ and ProTaper (62\%) $(p>0.05)$ ( - Fig. 2A).

\section{Discussion}

This study compared the cleaning efficacy of two different rotary NiTi instruments, RaCe and ProTaper Gold, in an experimental biofilm model (-Fig. 3). The model used in this study includes a multispecies mature biofilm grown on the endodontic system of freshly extracted monoradicular teeth. Part of the biofilm cultivation incorporated a starvation phase to increase the resistance and adherence of the biofilm. ${ }^{21-23}$ This starvation phase is essential in producing a biofilm, which more accurately represents an in vivo scenario. This begins to increase the translatability of the results from an in vitro model to an in vivo situation. Furthermore, the multispecies approach improves the standard of single-species infection that has been used traditionally in biofilm-based studies. Another advantage of the experimental model used in this study is the ability to separately assess the bacterial presence in the coronal, middle, and apical third of the root canal.

No files fractured during use in this study. The files possess different designs. The ProTaper system has a variable taper with an F2 file at a tip size of ISO 25 and an apical taper of $8 \%$, and the RaCe file has a tip size of ISO 25 and a taper of $5 \%$. The RaCe 123 system consists of three instruments; in our study, we used just two rotary RaCe NiTi files (T1, T2) in comparison with four ProTaper Gold files (S1, S2, F1, and F2). The current trend from manufacturers is to reduce and simplify the existing endodontic NiTi instruments
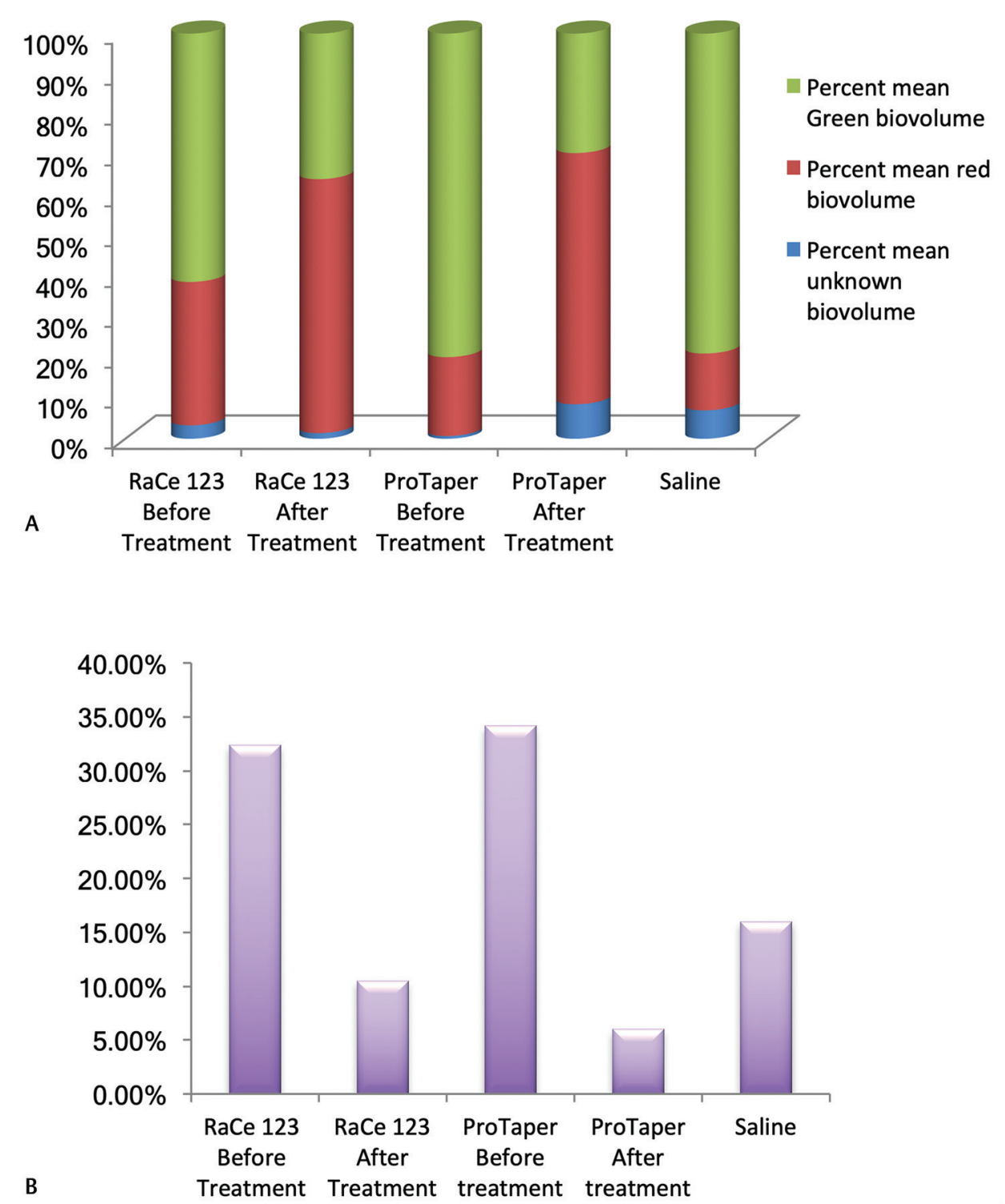

Fig. 2 Confocal laser scanning microscope analysis of the effect of treatment on multispecies biofilm models. (A) The effect of selected treatments on the mean percentages of dead (red), live (green), and unknown (blue) biovolumes of the biofilms in their respective treatment groups. (B) The effect of treatment on the mean percentage of substratum coverage of the biofilms. 


\section{Specimen Preparation}

$\mathrm{N}=39$

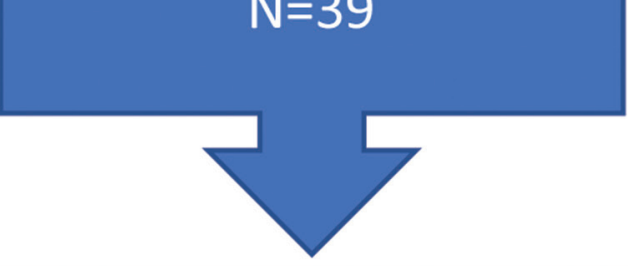

\section{Biofilm Development}

\section{Instrumentation and sampling} Quantitative viable count $(\mathrm{N}=24)$

$$
\text { A: RaCe } 123(n=9)
$$

B: Protaper Gold $(\mathrm{n}=9)$

$$
\begin{aligned}
& C=\text { Saline }(n=3) \\
& D=\text { Control }(n=3)
\end{aligned}
$$

\section{Confocal Laser Scanning Microscope (CLSM) Analysis of biofilm structure $(N=15)$}

$$
\begin{gathered}
\text { Group A (RaCe 123, } n=5) \text {, } \\
\text { Group B (ProTaper, } n=5) \\
\text { Group C (Saline, } n=5)
\end{gathered}
$$

\section{Results}

Fig. 3 Flow diagram showing the research methodology. 
sequences. ${ }^{24}$ The sizes of the instruments of the suggested RaCe 123 sequence are as follows: T1, 10/.06; T2, 25/.05; and T3 35/.04. A limitation within this study was the need for apical tip size standardization. The T3 file was omitted from the study to allow standardization and direct comparison with the F2 ProTaper Gold file at the apical level. Also, the taper of the compared instruments differs with a final taper of $5 \%$ for the RaCe sequence versus $8 \%$ variable taper for the ProTaper Gold. Clearly, the sampling procedures performed in this study could not evaluate the presence of bacteria within dentinal tubules.

This study demonstrates that even though differences in file designs exist, both ProTaper Gold and RaCe considerably reduced the number of microorganisms present in the root canal space compared with the uninstrumented control. The mechanism of bacterial reduction of mechanical shaping possibly involves the physical scraping of the biofilm adhering to the root canal walls. Once the biofilm is removed, it might be compacted in the flutes of the rotary instruments and hence removed from the root canal. Similarly to previous reports, neither file system rendered the canals bacteria-free. ${ }^{25}$ This is to be expected as it has been shown that mechanical cleaning alone may reduce intracanal bacteria, but it has not been possible to eliminate bacteria completely in this way. ${ }^{6,26,27}$ Mechanical cleaning coupled with irrigation is known to aid the removal of unwanted organic and inorganic material from the root canal system by flushing the loose debris..$^{20,25,26,28,29}$ Saline used with conventional syringe irrigation has proved its biofilm disruption effects. This is consistent with the previous studies. ${ }^{16}$ Therefore, the use of an irrigant coupled with the mechanical instrumentation would have acted as a confounder in the evaluation of the specific contribution of the instruments and the shaping components in the endodontic treatment. Further studies are planned that will include irrigation protocols combined with the tested instrumentation techniques and a control group left uninstrumented and exposed solely to the irrigant. To date, a limited number of papers have evaluated directly the efficacy of instrumentation per se in reducing the bacterial level. ${ }^{25,26}$ An average reduction of 1 log suggests the significant contribution of the mechanic instrumentation in reducing the bioburden present within the canal.

Given that there is no statistical difference across all positions within the root canal between the ProTaper Gold and RaCe 123 endodontic systems, it would seem that a reduced file sequence is an effective system at reducing bacterial load in a multispecies mature biofilm in an in vitro model; additional studies are needed to assess the cleaning efficacy of the numerous single-instrument techniques that are now being introduced in the market.

\section{Conclusions}

The use of ProTaper Gold and the RaCe 123 instruments on a nutrient-stressed multispecies biofilm in an in vitro root canal model resulted in significant bacterial killing and biofilm disruption rivaled with the uninstrumented control. There was no significant difference between the efficacy of
ProTaper Gold and the RaCe 123 instruments at any position in the root canal in terms of intracanal bacterial killing and biofilm disruption, demonstrating that the two tested systems were equally and solely effective in reducing microorganism within the endodontic space.

\section{Funding}

None.

\section{Conflict of Interest}

None declared.

\section{References}

1 Kakehashi S, Stanley HR, Fitzgerald RJ. The effects of surgical exposures of dental pulps in germ-free and conventional laboratory rats. Oral Surg Oral Med Oral Pathol 1965;20:340-349

2 Möller AJ, Fabricius L, Dahlén G, Ohman AE, Heyden G. Influence on periapical tissues of indigenous oral bacteria and necrotic pulp tissue in monkeys. Scand J Dent Res 1981;89(6):475-484

3 Bergenholtz G. Micro-organisms from necrotic pulp of traumatized teeth. Odontol Revy 1974;25(4):347-358

4 Schilder H. Cleaning and shaping the root canal. Dent Clin North Am 1974;18(2):269-296

5 Byström A, Sundqvist G. Bacteriologic evaluation of the efficacy of mechanical root canal instrumentation in endodontic therapy. Scand J Dent Res 1981;89(4):321-328

6 Dalton BC, Orstavik D, Phillips C, Pettiette M, Trope M. Bacterial reduction with nickel-titanium rotary instrumentation. J Endod 1998;24(11):763-767

7 Coffae KP, Brilliant JD. The effect of serial preparation versus nonserial preparation on tissue removal in the root canals of extracted mandibular human molars. J Endod 1975;1(6):211-214

8 Hsieh YD, Gau CH, Kung Wu SF, Shen EC, Hsu PW, Fu E. Dynamic recording of irrigating fluid distribution in root canals using thermal image analysis. Int Endod J 2007;40(1):11-17

9 Donlan RM, Costerton JW. Biofilms: survival mechanisms of clinically relevant microorganisms. Clin Microbiol Rev 2002;15(2):167-193

10 Costerton JW, Stewart PS, Greenberg EP. Bacterial biofilms: a common cause of persistent infections. Science 1999;284(5418):1318-1322

11 Bhuva B, Patel S, Wilson R, Niazi S, Beighton D, Mannocci F. The effectiveness of passive ultrasonic irrigation on intraradicular Enterococcus faecalis biofilms in extracted single-rooted human teeth. Int Endod J 2010;43(3):241-250

12 Prati C, Foschi F, Nucci C, Montebugnoli L, Marchionni S. Appearance of the root canal walls after preparation with NiTi rotary instruments: a comparative SEM investigation. Clin Oral Investig 2004;8(2):102-110

13 Molander A, Caplan D, Bergenholtz G, Reit C. Improved quality of root fillings provided by general dental practitioners educated in nickel-titanium rotary instrumentation. Int Endod J 2007;40(4):254-260

14 Gergi R, Rjeily JA, Sader J, Naaman A. Comparison of canal transportation and centering ability of twisted files, Pathfile-ProTaper system, and stainless steel hand K-files by using computed tomography. J Endod 2010;36(5):904-907

15 Pasqualini D, Bianchi CC, Paolino DS, et al. Computed micro-tomographic evaluation of glide path with nickel-titanium rotary PathFile in maxillary first molars curved canals. J Endod 2012;38(3):389-393

16 Niazi SA, Clark D, Do T, et al. The effectiveness of enzymic irrigation in removing a nutrient-stressed endodontic multispecies biofilm. Int Endod J 2014;47(8):756-768 
17 Niazi SA, Clarke D, Do T, Gilbert SC, Mannocci F, Beighton D. Propionibacterium acnes and Staphylococcus epidermidis isolated from refractory endodontic lesions are opportunistic pathogens. J Clin Microbiol 2010;48(11):3859-3869

18 Dahlén G, Samuelsson W, Molander A, Reit C. Identification and antimicrobial susceptibility of enterococci isolated from the root canal. Oral Microbiol Immunol 2000;15(5):309-312

19 Patel S, Wilson R, Dawood A, Foschi F, Mannocci F. The detection of periapical pathosis using digital periapical radiography and cone beam computed tomography - part 2: a 1-year post-treatment follow-up. Int Endod J 2012;45(8):711-723

20 Chávez de Paz LE. Image analysis software based on color segmentation for characterization of viability and physiological activity of biofilms. Appl Environ Microbiol 2009;75(6):1734-1739

21 Shen Y, Stojicic S, Haapasalo M. Bacterial viability in starved and revitalized biofilms: comparison of viability staining and direct culture. J Endod 2010;36(11):1820-1823

22 Liu H, Wei X, Ling J, Wang W, Huang X. Biofilm formation capability of Enterococcus faecalis cells in starvation phase and its susceptibility to sodium hypochlorite. J Endod 2010;36(4):630-635

23 Portenier I, Waltimo T, Ørstavik D, Haapasalo M. The susceptibility of starved, stationary phase, and growing cells of Enterococcus faecalis to endodontic medicaments. J Endod 2005;31(5):380-386
24 Bürklein S, Hinschitza K, Dammaschke T, Schäfer E. Shaping ability and cleaning effectiveness of two single-file systems in severely curved root canals of extracted teeth: Reciproc and WaveOne versus Mtwo and ProTaper. Int Endod J 2012;45(5):449-461

25 Pataky L, Iványi I, Grigár A, Fazekas A. Antimicrobial efficacy of various root canal preparation techniques: an in vitro comparative study. J Endod 2002;28(8):603-605

26 Shuping GB, Orstavik D, Sigurdsson A, Trope M. Reduction of intracanal bacteria using nickel-titanium rotary instrumentation and various medications. J Endod 2000;26(12):751-755

27 Chuste-Guillot MP, Badet C, Peli JF, Perez F. Effect of three nickel-titanium rotary file techniques on infected root dentin reduction. Oral Surg Oral Med Oral Pathol Oral Radiol Endod 2006;102(2):254-258

28 Siqueira JF Jr, Lima KC, Magalhães FA, Lopes HP, de Uzeda M. Mechanical reduction of the bacterial population in the root canal by three instrumentation techniques. J Endod 1999;25(5):332-335

29 Siqueira JF Jr, Rôças IN, Santos SR, Lima KC, Magalhães FA, de Uzeda M. Efficacy of instrumentation techniques and irrigation regimens in reducing the bacterial population within root canals. J Endod 2002;28(3):181-184 